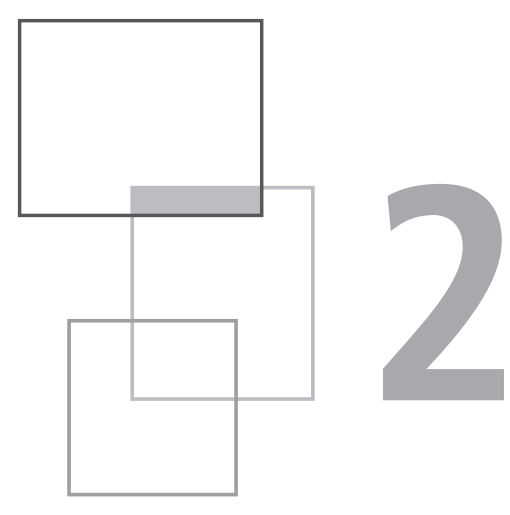

\title{
Services d'information
}

\subsection{Une raison d'être des services d'information}

L'intelligence d'information $(\$ 1.3)$ conduit à repenser l'approche à suivre pour surmonter les défis de la progression de la Société. Cette approche a pour intention de permettre à des groupes de personnes d'explorer et de construire ensemble des services d'information en établissant des consensus d'information et en partageant les responsabilités. Tous ces groupes sont hétérogènes dans le sens où les personnes les composant proviennent de différents métiers, de différentes entreprises publiques, privées, associatives ou internationales, ont des savoirs hétérogènes et assument des responsabilités diverses. Ainsi, les services concernés, dans leur sens le plus général, sont des trans-services dans le sens qu'ils sont transdisciplinaires, trans-institutionnels et même parfois transnationaux du fait de l'hétérogénéité de leurs contributeurs, qu'ils n'appartiennent exclusivement à aucune des disciplines, des institutions ou des nations contributrices, mais néanmoins qu'ils contribuent eux-mêmes à la propulsion de chacune des disciplines, des institutions ou même des nations concernées.

L'intelligence d'information relève les défis de l'expansion du champ des activités de la Société en conduisant des propulsions informationnelles. L'approche informationnelle suivie offre à un groupe hétérogène de personnes une cohésion cognitive jamais connue auparavant autour de services d'information. Elle l'amène à considérer que ses investigations doivent être essentiellement centrées sur des innovations d'information pour être consistantes face aux défis relevés. C'est ainsi que cette approche le conduit à lancer des processus de co-création et de co-conception de services d'information. C'est ainsi qu'il construit des services d'information comme premiers résultats. Ces résultats ne sont pas simplement des visions ou des rapports. 
Ils sont actionnables dans le sens que les acteurs, même ceux qui n'ont pas participé à ces explorations, peuvent les manipuler, non seulement comprendre mais actionner leur apport à leurs propres activités, les critiquer en sachant que leurs critiques seront débattues.

Les investigations rentrent alors dans une autre phase, celle de tenir compte des critiques. Dans ce but, elles sont amenées à faire évoluer les services d'information. Puis, avec les acteurs, d'une part, elles mettent en place leur exploitation et, d'autre part, elles organisent l'expansion des activités consécutives à l'exploitation de ces services d'information dans les différentes institutions, les différents métiers et disciplines ou les différents pays concernés.

Cette approche informationnelle considère les services d'information pour leurs potentialités d'offrir des activités beaucoup plus vastes que les traditionnels services du monde public ou privé, comme les services clients ou les services publics, ou du monde numérique comme les services Web. Elle les inscrit dans le cadre des propulsions informationnelles $(\$ 1.2 .5)$. Aussi au cœur de leur conception se trouvent, d'une part, leur composante numérique, contrairement aux services traditionnels de management, et, d'autre part, leur proposition de valeur dans la Société contrairement à un service habituel numérique.

Mais le fait qui rend les services d'information essentiels aux propulsions informationnelles, est la place centrale que l'information occupe dans tout service d'information. Un service d'information contient un socle informationnel avec des modèles informationnels $(\$ 1.2 .1)$ des connaissances sociétales et d'activités, des connaissances numériques et régulatoires. De plus, une fois le service d'information mis en exploitation, sa réalisation constitue son infrastructure informationnelle dans la Société avec ses aspects numériques, techniques et organisationnels.

Cette approche informationnelle est construite pour que les talents en intelligence d'information puissent faire preuve de leur esprit critique, se sentent libres de s'exprimer et de créer des services d'information à forte répercussion sur des expansions d'activités et ainsi contribuer à la propulsion informationnelle de la Société.

\subsection{Intention d'un service d'information}

Les bâtisseurs d'un service d'information ont l'intention de contribuer à surmonter une situation critique de progression de la Société qui concerne plusieurs secteurs de la Société.

Ils dressent les enjeux concernant leurs institutions, leurs entreprises, leurs professions ainsi que les formations à ces professions, leur organisation des activités et des responsabilités, leurs processus de gestion et de décision. Cette liste des enjeux sert de référence continuelle tout au long de la construction du service d'information.

Ils proposent des explorations en vue de construire un socle informationnel avec son infrastructure. Ce socle permet l'émergence d'activités du service en vue de surmonter la situation décrite dans l'intention. 
Ils formulent une proposition de valeur, qui explique comment le service va contribuer à l'expansion des activités de la Société et ainsi apporter de la valeur à la Société. Cette proposition de valeur présente aussi une estimation des chances et des risques à éviter dans l'exploration et la réalisation du service mais aussi les risques de ne pas entreprendre l'exploration et la réalisation du service d'information. Elle explore les modèles de toutes ces activités.

Ainsi, cette intention fournit un repère à tous les contributeurs du service et facilite ainsi leur unité cognitive. Elle est aussi un moyen de présenter l'identité cognitive du service aux partenaires extérieurs. Elle est au centre de la détermination du sens du service. L'intention, avec la situation visée, sa proposition de valeurs, ses enjeux, est complétée et affinée au fur et à mesure des explorations et de leurs découvertes ainsi que de la construction du service d'information.

\subsection{Canevas des activités autour d'un service d'information}

Toute personne assumant ses activités au travers d'un service d'information y retrouve de l'information mais aussi en apporte de nouvelles, en modifie d'autres ou en rend d'autres obsolètes. Elle contribue au service d'information en bénéficiant et en fournissant de l'information au service d'information, même si, dans des cas particuliers, elle tient seulement l'un des deux rôles soit bénéficiaire soit fournisseur comme dans le cas des services habituels.

Les contributeurs d'un service d'information sont des acteurs ou des bâtisseurs. Les acteurs exercent des activités en remplissant des responsabilités ou en exécutant des tâches, au travers du service d'information : leurs activités sont exogènes au service d'information. Les bâtisseurs exercent des activités concernant directement le service d'information, comme celles de sa création, conception, réalisation, opérationnalisation, maintenance, évolution : leurs activités sont endogènes au service d'information. Un contributeur peut être à la fois acteur et bâtisseur.

\subsubsection{Cadre de référence des contributeurs}

Tout service d'information établit une sorte de nouvelle ville construite sur le numérique avec de nouveaux enjeux, de nouveaux usages et de nouvelles formes de relations humaines entre tous les contributeurs, bâtisseurs et acteurs. Il leur faut des repères dans ces situations nouvelles pour adopter des attitudes et des comportements fluides en concordance avec les autres contributeurs et pour établir la confiance avec les autres de manière durable.

Ces repères sont formalisés sous forme de protocoles, c'est-à-dire un ensemble de règles et d'usages à observer dans leurs relations avec les autres contributeurs au travers du service d'information. Ces règles et usages concernent par exemple les 
modes de partage d'informations, de coordination, de co-création des extensions du service et même de co-création de nouveaux services. Elles peuvent avoir des bases légales, réglementaires ou éthiques.

Ces protocoles concernent toutes les activités s'exerçant au travers du service. Parmi ces protocoles, il y a ceux qui régissent les recouvrements informationnels entre différentes activités : une situation de recouvrement informationnel apparaît dès qu'au moins deux activités non seulement partagent des mêmes informations mais aussi les mettent à jour. Dans cette situation, il faut mettre en place des protocoles pour déterminer les rôles de chacune des activités concernées afin de maintenir la cohérence informationnelle du service d'information comme dans Léonard et Parchet (1999) et Snene et Léonard (2009).

Le cadre de référence des contributeurs d'un service d'information est composé de tous ces protocoles.

\subsubsection{Administration d'un service d'information}

L'administration d'un service d'information concerne le respect de ce cadre de référence pour permettre le bon fonctionnement du service d'information. Elle prend les mesures opérationnelles appropriées. Ainsi, elle s'assure du respect des protocoles par les contributeurs, de la conformité des informations apportées par les contributeurs aux éléments régulatoires du service. Elle prend aussi toutes les mesures pour assurer ou améliorer le bon fonctionnement du service comme celles pour améliorer l'ergonomie, ou pour augmenter des performances, ou pour apporter des modifications aux protocoles d'échanges, d'accès aux informations, de coordination entre les contributeurs.

Elle assure aussi toutes les activités d'alimentation du service en informations nécessaires au bon fonctionnement des activités exogènes du service comme les informations régulatoires et elle les maintient à jour. Elle contrôle également la conformité des activités exogènes avec le monde régulatoire du service.

\subsubsection{Pilotage d'un service d'information}

Le pilotage d'un service d'information s'occupe de l'avenir du service d'information, sa création et sa progression. Il est placé sous la direction d'un comité de pilotage. Les activités de pilotage sont assumées par des bâtisseurs du service.

\subsubsection{Veille}

Le pilotage d'un service d'information demande des informations concernant les situations dans lesquelles les contributeurs du service exercent leurs activités, qu'ils soient acteurs ou bâtisseurs et les transformations de l'environnement du service lui-même comme des textes régulatoires et de ses activités exogènes. Ce sont des activités de veille qui collectent ces informations. 
Ainsi certaines d'entre elles détectent dans les activités des contributeurs, des faiblesses cognitives par manque d'information, des surcharges cognitives, des mauvaises performances, ou bien toutes sortes de difficultés rencontrées par les contributeurs par exemple dans leurs échanges ou leurs coordinations. Elles analysent leurs origines et prennent les décisions pour y remédier en fonction de l'avenir du service, comme mettre en place une nouvelle formation, un nouvel espace d'apprentissage, demander des modifications ou penser à une évolution du service.

D'autres activités de veille observent l'environnement du service d'information et de ses activités exogènes pour détecter des opportunités d'alimenter le service avec de nouvelles informations, d'étendre le champ des activités des contributeurs ou d'en créer de nouvelles, d'ouvrir le service à d'autres contributeurs. Après avoir conduit leur investigation, ces activités conduisent à des décisions pour des affinements ou même des modifications de l'intention du service, de sa proposition de valeur ou de son sens. Ces décisions peuvent même ouvrir le champ à des explorations de son avenir.

La veille d'un service d'information concerne aussi les échanges entre les contributeurs : prendre en compte les difficultés relationnelles qui peuvent surgir dans les échanges ou au contraire dans l'absence d'échanges, en trouver les origines qui proviendraient du service lui-même ou de son administration, et ensuite prendre les mesures adéquates. Ces activités doivent se mêler aux activités des contributeurs et leur devenir indispensables, et alors elles apparaissent comme un mélange d'activités de concierge, d'accueil et d'animation.

\subsubsection{Création et progression d'un service d'information}

Les activités de pilotage concernent la création ou la progression d'un service d'information. Notamment, elles fournissent des réponses aux situations soulevées par les situations mises en évidence par les activités précédentes.

Les bâtisseurs de service mettent en place les modèles du socle informationnel du service. Ils les confient aux activités de réalisation pour rendre opérationnel le service. Ils confient aux activités d'alimentation du service la constitution de toutes les informations régulatoires. Ils établissent l'intention du service avec sa proposition de valeur, le premier cadre de référence des contributeurs. Ils font la promotion du service pour attirer des contributeurs.

La structure d'un service n'est pas inerte ; elle doit progresser par exemple pour faire face à de nouvelles attentes des contributeurs, pour intégrer de nouvelles technologies de l'information ou des modifications régulatoires. Ce sont de nouveau des bâtisseurs qui impulsent cette progression. Ils font le même genre de travail que lors de la création mais ils doivent tenir compte du fait que le service est actif et que des contributeurs exercent déjà leurs activités avec lui et ne peuvent généralement pas les arrêter pour attendre la nouvelle version du service.

Avec la coopération des contributeurs concernés, ils font progresser le service en faisant évoluer les modèles, en enrichissant l'intention du service, le cadre de référence des contributeurs, et en fin de compte en proposant une nouvelle version du service. 
Ils participent à la décision finale avec le comité de pilotage. En cas de réponse positive, ils rendent opérationnelle la nouvelle version du service et débranche celle qui devient obsolescente.

\subsubsection{L'histoire du service}

Les activités relatives à l'histoire du service sont exercées par des bâtisseurs tenant un rôle spécial : ils n'ont pas un rôle actif dans la création et la progression d'un service d'information, tout en faisant partie intégrante des équipes des bâtisseurs qui conduisent les explorations. La mission de ces activités est de relater l'histoire du service au fur et à mesure des explorations et des réalisations. En particulier, elles sont en charge de la conservation de tous les documents fondamentaux des bâtisseurs, comme l'intention, le cadre de référence des contributeurs dans leurs différentes versions. Elles doivent tenir à jour le tableau d'honneur du service qui comprend notamment le tableau d'honneur des contributeurs où elles inscrivent pour chacun d'eux leurs contributions au service, et celui des événements significatifs de la progression du service. Elles doivent être les gardiennes du sens du service.

Leur rôle est primordial pour conserver l'unité et l'identité cognitives de tous les contributeurs du service et pour la formation de nouveaux contributeurs.

\subsubsection{Synthèse des activités autour d'un service d'information}

La figure 2.1 fait la synthèse de toutes ces activités.

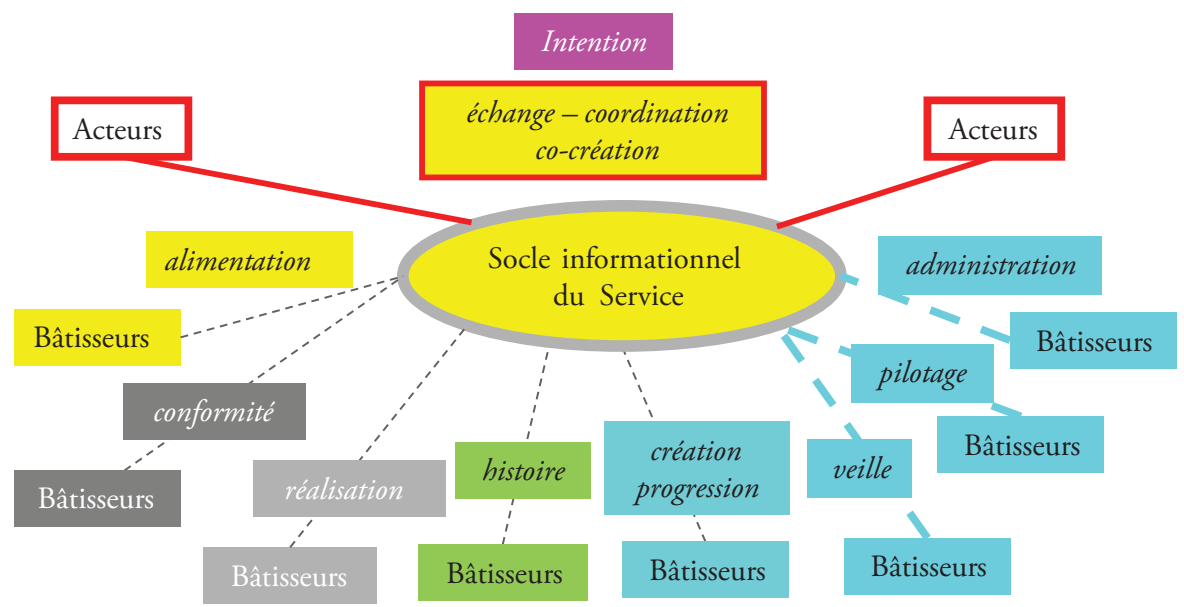

Figure 2.1. Activités autour d'un service. 


\subsubsection{Canevas d'un service d'information}

Le canevas d'un service d'information comprend six dimensions (figure 2.2) :

- intention ;

- cadre de référence des contributeurs ;

- socle informationnel ;

- histoire ;

- infrastructure informationnelle ;

- espace opérationnel.

Les quatre premières dimensions viennent d'être présentées.

La dimension de l'infrastructure informationnelle concerne tous les aspects numériques, techniques et organisationnels de la mise en œuvre du service dans la Société : par exemple, tous les systèmes numériques utilisés pour construire la partie numérique du service, tous les équipements pour remplir certaines activités exogènes, et aussi la réorganisation éventuelle de bureaux.

La dimension de l'espace opérationnel d'un service d'information concerne toutes les activités s'exerçant autour du service, comme celles de sa création, de sa progression, de son alimentation, mais aussi les activités opérationnelles des services exogènes construits à partir de lui.

L'espace opérationnel d'un service d'information est la conséquence directe des activités de sa création et de sa progression. Il doit être continuellement en arrière-fond de toutes leurs explorations et conceptions.

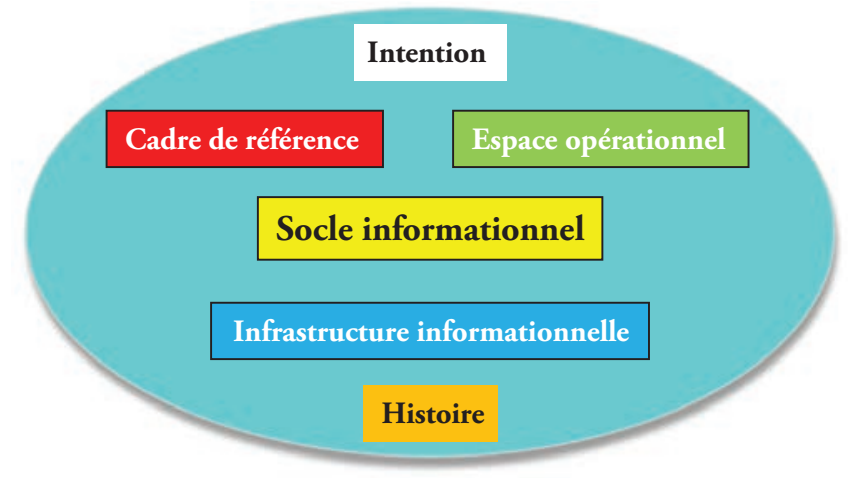

Figure 2.2. Canevas d'un service.

Le canevas d'un service d'information est affiné tout au long des processus de création et de réalisation. 


\subsection{Service complexe d'information}

Une propulsion informationnelle demande plus qu'un ensemble de services dispersés. Il lui faut des services complexes appelés des systèmes de services obtenus à partir d'autres services, ses services de base : " un système de services est une configuration de ressources pour une co-création dynamique de valeurs, ces ressources comprenant des personnes, des organisations, des informations partagées (langage, lois, mesures, méthodes) et des technologies, toutes connectées intérieurement et extérieurement à d'autres systèmes de services au travers de propositions de valeurs " (Maglio et al., 2009).

Un service complexe d'information est un service d'information obtenu par composition de services d'information, qui sont ses services de base. C'est ainsi un système de services.

Cette composition concerne les relations entre le canevas propre du service complexe d'information et les canevas de ses services d'information de base.

\subsubsection{Principes de base de la composition de services}

Un service complexe est une composition de services et son canevas doit être établi comme une composition des canevas des services de base. Cette composition permet de clarifier les relations entre le service complexe et ses services de base. Elle permet de mettre en évidence un ensemble de situations génériques, cruciales et déterminantes que les processus de construction de service complexe peuvent rencontrer :

- génériques : elles ne dépendent d'aucun domaine particulier ;

- cruciales : elles demandent une décision de conception irréversible ;

- déterminantes : leurs conséquences sont importantes ; elles ne peuvent pas être prises en compte si, par exemple, on ignore leur existence, et alors, inévitablement, l'ampleur de leurs conséquences ne peut être examinée et sera du ressort de la main invisible $(\$ 1.1 .4 .3)$.

\subsubsection{Types d'alliance dans un service complexe}

Un service complexe constitue une alliance entre lui-même et ses services de base et aussi une alliance entre les services de base eux-mêmes. Ce paragraphe en présente cinq types. Chacun est lié à un mode de composition des canevas des services de base pour former le canevas du service complexe. Il a des conséquences sur les modes de fonctionnement des services de base et sur les rôles de leurs contributeurs.

\subsubsection{Intégration}

Tous les services de base sont intégrés dans le service complexe. Leur intention n'a plus lieu d'exister. Par contre l'intention du service complexe doit les reprendre en 
tenant compte de la nouvelle dimension prise par cette alliance pour répondre à des enjeux sociétaux.

Les acteurs de tous les services de base deviennent des acteurs du service complexe: ils continuent à exercer leurs activités exogènes dans des conditions informationnelles identiques.

Toutes les activités de pilotage et d'administration effectuées au niveau des services de base sont intégralement assumées au niveau du service complexe. Les cadres de référence des contributeurs des services de base n'ont plus de raison d'exister.

Le socle informationnel, l'infrastructure informationnelle et l'espace opérationnel du service complexe engloutissent ceux des services de base.

L'histoire des services de base s'arrête, celle du service complexe commence.

\subsubsection{Centralisation}

Toutes les activités de pilotage des services de base sont centralisées au niveau du service complexe. Le socle informationnel, l'infrastructure informationnelle et l'espace opérationnel du service complexe engloutissent ceux des services de base.

Tous les services de base conservent leur intention, leurs acteurs et leur cadre de référence qui ne concernent plus que leur administration ainsi que l'administration des activités exogènes. Leurs bâtisseurs s'occupent seulement de l'administration. Leur histoire continue.

\subsubsection{Orchestration}

Toutes les activités de pilotage des services de base sont approuvées et supervisées au niveau du service complexe. Un service de base conserve toutes ses activités endogènes et exogènes, ses bâtisseurs et ses acteurs, son cadre de référence des contributeurs, son socle informationnel, son infrastructure informationnelle, son espace opérationnel. Son histoire continue.

Mais il est soumis aux activités transversales décidées au niveau du service complexe. Ces activités transversales s'exercent sur plusieurs services de base par des acteurs du service complexe. Leurs bâtisseurs sont des contributeurs du service complexe. Ils doivent compter sur la coopération des bâtisseurs des services de base pour faire progresser leur service afin de permettre aux activités transversales de s'exercer sur les infrastructures informationnelles des services de base. Le cadre de référence d'un service de base doit être étendu pour prendre en compte bâtisseurs et acteurs d'activités transversales.

Pour le système complexe, l'intention fait référence aux intentions des services de base, mais elle se concentre particulièrement sur les activités transversales et leurs propositions de valeur.

Le cadre de référence comprend un protocole pour administrer les activités transversales : il concerne les relations entre les comités de pilotage du service complexe 
et des services de base concernés dès la phase d'exploration. Il doit gérer les relations entre les bâtisseurs et acteurs du système complexe et les bâtisseurs et acteurs des services de base concernés. Il doit établir des protocoles de recouvrements informationnels entre les différents services de base traversés par des activités transversales.

Le socle informationnel du service complexe contient toutes les informations nécessaires à son administration et à son pilotage. De plus, compte tenu des activités transversales, il est étendu à tout l'espace informationnel des activités transversales, c'est-à-dire l'ensemble des informations et des traitements auxquels elles accèdent (\$1.2.3), y compris dans les socles informationnels des services de base. Il en est de même pour l'infrastructure informationnelle.

L'espace opérationnel et l'histoire, en plus de leur rôle habituel, tiennent compte des événements importants qui surviennent aux services d'une orchestration, comme le lancement d'une exploration d'extension d'un service de base qui a été approuvé.

\subsubsection{Chorégraphie}

Toutes les activités de pilotage des services de base d'une chorégraphie sont soumises au comité de pilotage du service complexe non pas pour qu'il les approuve et ensuite les supervise, mais pour qu'il garantisse aux autres parties de la chorégraphie leur conformité à l'esprit de la chorégraphie.

Cet esprit de la chorégraphie s'exprime dans l'intention des différents services de base de se constituer en une chorégraphie, de pouvoir créer des activités transversales et d'avoir décidé des modes de règlement de divergences cognitives concernant la progression de l'un d'entre eux ou celle d'une activité transversale. Ces divergences sont débattues au niveau de la chorégraphie.

Mais ce n'est pas au niveau de la chorégraphie que sont prises les décisions d'explorer une activité transversale, puis de la réaliser, puis de la rendre opérationnelle. Ce sont les comités de pilotage des services de base qui assurent ces responsabilités. Les bâtisseurs de ces activités transversales sont des bâtisseurs des services de base concernés. Il en est de même pour les acteurs.

Le service complexe se définit à l'aide de son intention, de son cadre de référence qui s'impose comme le noyau des cadres de référence des services de la chorégraphie, et de son espace opérationnel pour les activités transversales.

\subsubsection{Farandole}

Les bâtisseurs d'une farandole sont des bâtisseurs de services d'information qui vont la composer. Ils ont l'intention de créer une base commune de toutes leurs activités concernant leur identité et leur unité cognitives pour faciliter la mise en place d'accords de coopération. Ces accords portent par exemple sur l'extension d'une activité d'un service à un autre service, dans le sens où cette activité va pouvoir traiter des informations de l'autre service. Un accord de coopération ne concerne pas obligatoirement tous les services composant la farandole. 
Le canevas du service complexe formé à partir d'une farandole, contient seulement l'intention et le cadre de référence des contributeurs des services de base. L'intention est celle des bâtisseurs de la farandole. Le cadre de référence précise la base commune qui se retrouve dans les canevas de tous les services de la farandole : ainsi le cadre de référence de tout service de la farandole doit contenir un protocole pour établir une coopération avec les autres services de la farandole.

Les activités transversales évoquées dans une orchestration et dans une chorégraphie sont prises en compte d'une toute autre manière dans une farandole. Une activité transversale se met en place par des accords de coopération, en restant au seul niveau des services de base et non au niveau du service complexe.

\subsubsection{Situation cruciale et déterminante du choix du type d'alliance}

Les différences entre ces cinq types d'alliance, qui se dégagent des principes de leur fonctionnement, ont des répercussions importantes sur les canevas des services de base. La situation du choix du type d'alliance est cruciale et déterminante pour la construction du service complexe car il détermine les évolutions à apporter aux canevas des services de base.

Il faut explorer les différents types d'alliance et en évaluer les répercussions, sans oublier le maintien en activité des activités exogènes des services de base. Le choix décidé sera difficile à remettre en cause ensuite.

Cette exploration ne doit surtout pas se limiter aux seules considérations managériales ou numériques. Elle doit surtout analyser avec soin les socles informationnels des différents services de base et se concentrer sur leurs recouvrements informationnels.

Deux services ont un recouvrement informationnel (\$1.2.3) si elles disposent des informations d'un même phénomène - comme par exemple des règlements ou des lois. Cette situation du recouvrement informationnel conduit à une situation de redondance informationnelle qui est particulièrement préoccupante au moment de mises-à-jour pour garantir leur synchronisation. Or, cette situation de recouvrement est inhérente à la constitution d'une alliance entre des services d'information : en effet, dans le cas général, ils ont été construits séparément et fonctionnent indépendamment les uns des autres ; de plus ils ont forcément des recouvrements informationnels entre eux puisqu'on envisage de les composer. C'est souvent une raison importante de l'intention de constituer une alliance.

Mais la situation de recouvrement n'est pas simple. En effet, un recouvrement informationnel entre deux services induit une situation de redondance informationnelle, les deux services disposant d'informations relatives à un même phénomène - comme par exemple des règlements ou des lois. S'ils font partie d'une même alliance, alors il est indispensable de synchroniser les mises-à-jour pour que, par exemple, un troisième service de l'alliance obtienne la même réponse qu'il s'adresse à l'un ou l'autre des services. C'est tout un protocole de recouvrement qu'il faut établir. La difficulté de le mettre en place dépend fortement de l'implémentation 
des parties informationnelles du recouvrement dans les deux infrastructures informationnelles, tant au niveau numérique qu'au niveau organisationnel. Comme les infrastructures informationnelles ont été construites et réalisées séparément, la difficulté est généralement élevée, parfois impossible!

Cette situation de recouvrement est cruciale et déterminante dans le choix du type de l'alliance. Elle est cruciale car elle conditionne les méthodes numériques et organisationnelles pour l'implémenter dans l'alliance et assurer sa synchronisation. Elle est déterminante car si elle n'est pas connue, on ne peut qu'ignorer les enjeux d'implémentation des recouvrements et être inconscient des impossibilités numériques et des possibles conflits organisationnels.

\subsubsection{Extension de la définition d'un service}

Pour l'instant, un service d'information a un cadre défini sur lui-même. Ses activités sont internes, reposent entièrement sur son socle informationnel et s'exécutent sur son infrastructure informationnelle. L'enjeu de son cadre de référence est l'unité et l'identité cognitives de tous ses contributeurs. Les explorations pour son avenir ne concernent que ses activités.

Mais ce cadre de pensée est trop étriqué pour les services complexes. C'est comme si l'on considère une ville seulement pour ses habitants et pour les activités qu'ils y exercent, bien qu'elle fasse partie d'un réseau plus vaste avec des activités transversales, comme la poste. Il faut alors considérer aussi les personnes qui exercent ces activités transversales dans la ville, même s'ils ne sont pas des habitants.

Il faut étendre le cadre de pensée d'un service. Une alliance entre services, dans le cadre d'un service complexe, conduit à deux types d'extension de la définition d'un service et finalement à une extension des activités exogènes.

\subsubsection{Extension induite par la transversalité}

Une alliance de type orchestration, chorégraphie ou farandole, permet les activités transversales. Une activité transversale concernant un service de base repose son infrastructure informationnelle mais aussi sur celles d'autres services de base. Les acteurs de cette activité sont amenés à exercer leurs activités à l'aide du service de base, mais ils le font par intermittence, sans être impliqués par l'ensemble du service. Le cadre de référence du service doit être étendu à ces acteurs intermittents et à leurs activités. Il doit définir le cadre des échanges entre les acteurs intermittents et les acteurs permanents qui étaient les seuls acteurs pris en compte avant l'extension. Cette extension a des répercussions sur le socle informationnel et l'infrastructure informationnelle, tant au niveau numérique qu’au niveau organisationnel, par exemple pour garantir sûreté et sécurité informationnelle. Elle a aussi des répercussions sur l'espace opérationnel du service de base : les explorations doivent tenir compte des activités transversales. 


\subsubsection{Extension induite par les recouvrements informationnels}

La situation du recouvrement informationnel est pratiquement inévitable entre deux ou plusieurs services qui ont été construits indépendamment les uns des autres et qui donnent lieu à des activités transversales dans des alliances. Les difficultés à surmonter ont été présentées précédemment (\$2.4.2.6). Notamment elles concernent la redondance informationnelle et la synchronisation des mises-à-jour entre tous les services concernés. Une possibilité pour surmonter cette difficulté est de construire un nouveau service, un service de recouvrement dont le socle informationnel est formé à partir du recouvrement informationnel. Les mises-à-jour du recouvrement sont apportées au service de recouvrement et les autres services concernés les répercutent chez eux.

Les services concernés doivent alors constituer une alliance entre eux et avec ce service de recouvrement de type d'une chorégraphie ou d'une farandole. Leur socle informationnel est alors constitué de deux parties : le socle informationnel propre qui est leur socle initial et le socle informationnel virtuel qui est celui du service de recouvrement. Il en est de même de leur infrastructure informationnelle. Ainsi, le socle informationnel des services est étendu à des parties informationnelles qui se trouvent dans un autre service : en conséquence l'espace informationnel de leurs activités concernées par le recouvrement est aussi étendu. Leurs canevas doivent être conformes à cette extension.

De son côté, le service de recouvrement doit admettre comme acteurs intermittents tous les acteurs des activités exogènes concernées par le recouvrement.

\subsubsection{Extension des activités exogènes}

Les extensions précédentes d'un service ouvrent alors une possibilité lumineuse à toute activité exogène, celle d'étendre son espace informationnel à des informations gérées dans d'autres services. Elle devient ainsi une activité exogène étendue à ces autres services. Il suffit que tous ces services appartiennent à une même alliance pour régler toutes les situations causées par une telle extension : par exemple les acteurs de l'activité exogène d'un service deviennent aussi des acteurs intermittents des services concernés par son extension.

Cette possibilité d'extension de l'espace informationnel d'une activité exogène d'un service d'information n'est pertinente que si elle permet à l'activité exogène d'enrichir sa proposition de valeur. Cela implique que les bâtisseurs d'une activité exogène doivent explorer des évolutions non seulement en considérant l'intérieur de son service mais également en considérant les autres services appartenant à une même alliance.

Mais alors c'est la proposition de valeur de l'alliance elle-même qui est enrichie puisque c'est elle qui permet ces extensions d'activités exogènes des services qui la composent.

C'est une situation qu'il faut examiner avec attention au moment du choix du type d'alliance entre les services d'information : lequel de ces types permet-il le plus 
d'agilité pour de tels processus d'innovation en prenant en compte tous les niveaux de canevas des services concernés?

C'est une situation cruciale car une fois le type d'alliance décidé et mis en place, il sera pratiquement impossible de le remettre en cause. C'est une situation déterminante car elle peut être ignorée par manque de connaissances de son importance pour les possibilités ultérieures d'innovation.

Sans tenir compte d'autres facteurs, l'alliance de type farandole apparaît de manière générique la plus agile, et dans une moindre mesure celle de type chorégraphie aussi.

\subsubsection{Transformation d'un service en un service complexe}

Les paragraphes précédents ont montré comment il est possible de construire un service complexe par la composition de services existants. C'est une situation essentielle rencontrée dans les propulsions de la Société. Mais il en est une autre tout aussi essentielle, qui est l'inverse de la situation précédente : la décomposition d'un service en plusieurs services.

En effet, une propulsion peut conduire un service d'information à devenir de plus en plus imposant au fur et à mesure d'évolutions, pouvant être causées par le succès de ses activités exogènes. Alors son administration peut devenir lourde, de moins en moins efficiente, et surtout, il y a de moins en moins de bâtisseurs pour proposer et conduire des explorations afin de construire l'avenir du service. Il est devenu trop gros, il s'imprègne de stérilité cognitive, il devient de plus en plus un fardeau pour la progression de la Société.

Une possibilité est justement de le considérer comme formant un système complexe intégré dans un seul service. Il s'agit de le décomposer en plusieurs services dont l'alliance permet de le reconstituer par composition. La décomposition est l'opération inverse de la composition présentée précédemment.

Pour mener à bien cette décomposition, il s'agit de déterminer le plus tôt possible le type d'alliance entre centralisation, orchestration, chorégraphie et farandole. Le choix doit se faire en donnant une place centrale à la décomposition du socle informationnel et de son infrastructure informationnelle en faisant particulièrement attention aux recouvrements informationnels.

La décomposition du service initial conduit ainsi à le transformer en un service complexe dont les services de base sont les services obtenus par la décomposition. Les canevas de ces services doivent pouvoir former le canevas du service complexe par composition.

Une telle décomposition permet de retrouver de l'agilité notamment en redonnant des possibilités d'exploration au niveau des services obtenus par la décomposition. 


\subsection{Cohésion cognitive : espace de pollinisation croisée}

Dans l'approche informationnelle, c'est autour des services d'information que se met en place la cohésion cognitive ou au contraire la confusion cognitive entre les personnes concernées par la propulsion $(\$ 1.2 .6)$. La cohésion cognitive ne peut pas leur être imposée, elle ne peut être ni un résultat, ni une solution. Simplement les acteurs la ressentent notamment par la fluidité de leurs activités.

C'est ainsi que le processus de construction d'un service d'information proposé par l'approche informationnelle conduit à éviter d'importantes situations propices à induire de la confusion cognitive entre les contributeurs et ainsi à faciliter la mise en place d'une cohésion cognitive.

De manière générale, les bâtisseurs d'un service d'information forment un groupe hétérogène du point de vue de leurs métiers, de leurs responsabilités, de leurs institutions, pour construire un trans-service, un service d'information transdisciplinaire, trans-institutionnel et même parfois transnational $(\$ 2.1)$.

Même s'ils parlent la même langue maternelle, ils ne vont pas forcément se comprendre. En effet, chacun parle la langue de son métier ou de ses responsabilités, conduit des raisonnements propres à son métier ou à son institution, et ainsi rend les autres interloqués par l'usage de certains termes, par des expressions de métier incompréhensibles, et, finalement, les pousse dans la confusion.

Aussi ne suffit-il pas de les réunir autour d'une table pour leur permettre de co-créer un trans-service. L'approche informationnelle leur propose un langage commun, celui de l'information.

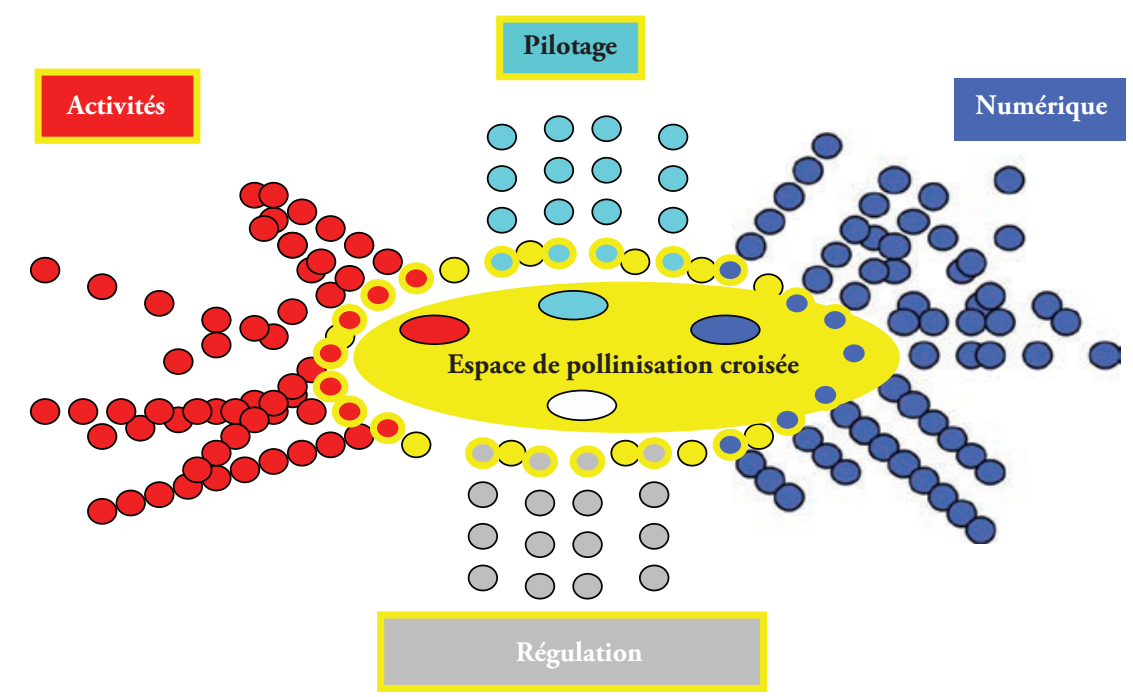

Figure 2.3. Espace de pollinisation croisée. 
Dans la figure 2.3, les cercles rouges symbolisent des personnes remplissant des activités (métiers, responsabilités, tâches) et ceux entourés de jaunes qui sont autour de la table symbolisent celles d'entre elles parlant le langage de l'information. Les cercles en turquoise, bleu, gris désignent des personnes dont les activités concernent respectivement le pilotage, le numérique et la régulation (connaissances, lois, standards...). Les cercles en jaune autour de la table symbolisent des spécialistes en langue de l'information.

C'est dans ce langage de l'information que sont établis tous les modèles de conception du service et ainsi ils peuvent être compris, discutés, améliorés par tous les bâtisseurs. C'est grâce à lui que sont exprimés les résultats des explorations et qu'ils sont rendus actionnables $(\$ 2.1)$.

C'est grâce à lui que certains bâtisseurs peuvent proposer des évolutions dont l'intérêt peut être partagé, critiqué, aménagé par les autres. C'est grâce à lui que certaines innovations provenant de bâtisseurs de certains domaines peuvent conduire à des innovations dans d'autres domaines.

C'est lui qui permet d'éviter des situations où certains bâtisseurs voudraient imposer leur solution en masquant leurs intentions et qui entraîneraient des confusions dans les évolutions du service. Il tient une place centrale dans l'approche informationnelle pour qu'une cohésion cognitive s'installe entre les bâtisseurs.

Cet espace de pollinisation croisée est aussi très intéressant pour les acteurs du service car il leur permet de mieux appréhender les entrelacements de leurs activités et de mieux saisir l'aide qu'ils peuvent attendre du service dans l'exercice de leurs activités (Yurchyshyna et al., 2011). Il devient une place centrale de leur cohésion cognitive.

Enfin, cet espace œuvre aussi à la cohésion cognitive entre bâtisseurs et acteurs d'un service. Il procure un espace d'échanges entre eux, notamment autour des résultats actionnables mis en place par les bâtisseurs. Il facilite le passage de la disruption cognitive des activités provoquée par les bâtisseurs en créant et réalisant le service, à la continuité cognitive indispensable aux acteurs pour assurer sereinement leurs activités $(\$ 1.2 .5 .5)$.

\subsection{Conclusion}

Ce chapitre vient de présenter les principales caractéristiques des services d'information. Ils constituent les piliers des propulsions informationnelles. Maintenant, il s'agit de décrire comment les mettre en action pour assurer la progression de la Société dans le cadre de propulsions informationnelles : ce sera l'objet du chapitre 4 Administration d'une propulsion informationnelle. Ensuite, dans le chapitre 5, il s'agit de dégager les premiers principes de l'intelligence de service pour assurer la progression de la Société. Mais avant, il s'agit de présenter un concept central dans la mise en action des services d'information dans des propulsions informationnelles, celui des communs informationnels : c'est l'objet du prochain chapitre. 